\title{
Experimental determination of the induction period for the onset of intensive boiling of a subcooled water flow under conditions of unsteady heat release
}

\author{
A. Safarov, A. Levin* \\ Melentiev Energy Systems Institute of Siberian Branch of Russian Academy of Sciences, Irkutsk, Russia

\begin{abstract}
This paper studies prediction of the boiling crisis onset under conditions of the rapid temperature growth of the heat releasing surface washed by a water flow subcooled to the saturation temperature. We obtained experimental data on time delay for rapid vapor formation and showed that the existing technique can be extended to the case with a forced flow. It is shown that the characteristic layer thickness is smaller than the thickness of superheated layer.
\end{abstract}

Index Terms - unsteady heat exchange, nucleate boiling, boiling crisis.

\section{Introduction}

The practical use of the boiling subcooled liquid is motivated by the possible achievement of high values of the heat flux. However, it is known that the stable nucleate boiling is bounded from above by a critical heat flux value, and when this value is reached we can observe a regeneration of vapor structures on the heat releasing surface. As the temperature grows, bubbles start to merge into large agglomerations and, once certain conditions are fulfilled, form a continuous vapor layer. The critical heat flux for many types of liquid has been studied for a long time under various conditions. However, the sharp change of heat power remains the least studied field in this area of research.

If we focus on mechanics of the boiling crisis onset in case of unsteady heat release [2-4], we can establish two important stages:

1. Heating of the near-wall liquid layers to the temperature required to form the first bubble. The prediction methods for this stage are developed quite well.

2. Development of nucleate boiling up to the moment of formation of rapidly growing vapor agglomerations. At this stage, we can observe a rapid increase of the number of vaporization centers on the surface $N_{a}$, an increase of the superheated layer thickness $\delta_{\mathrm{s}}$ and a growth of maximum bubble diameters $D_{m}$. A certain combination of $N_{a}$ and $D_{m}$ causes an avalanche-like increase of vapor volume, accompanied by an increase in pressure (Fig.1).

In this work, we experimentally determined the time $\tau_{\text {ind }}$ required for the second stage to begin under the conditions of the forced movement of the subcooled water flow.

\section{Experimental setup}

The experimental unit comprises a closed isolated circuit with a forced flow equipped with a system that ensures automatic maintenance of the required conditions (temperature, flow rates, liquid pressure). The unit scheme is presented at Fig. 2. The flow part of the circuit includes a channel with transparent walls and with a heater inside it. The heater is a stainless steel tube with an outer diameter of $12 \mathrm{~mm}$ and a wall thickness of $1 \mathrm{~mm}$. The maximum roughness of the heater's outer surface is $4 \mu \mathrm{m}$. The external surface of the heater is washed with moving water, and thermocouples are welded to the internal surface to measure metal temperature.

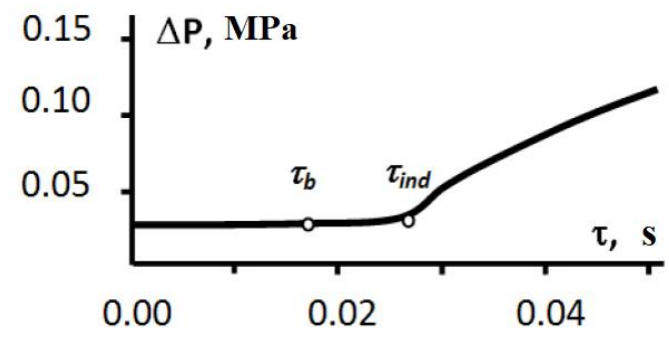

Fig. 1. Pressure growth dynamics in the channel after the start of heating

The circuit includes a heater and a cooler to set up an 
initial subcooling of water. The overpressure in the circuit is regulated by a separate device connected at the top. The pressure is varied by changing the temperature of water, which is in thermodynamic equilibrium with wet vapor. The tank for preliminary degassing of water is located at the bottom right corner.

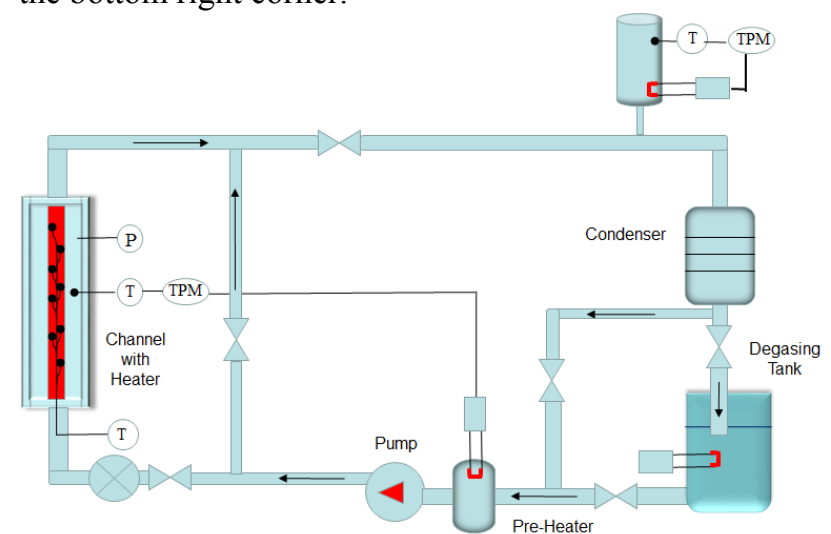

Fig. 2. Scheme of the experimental unit.

The experiments were carried out at an overpressure of $0.138 \mathrm{MPa}$, which corresponds to the saturation temperature of $102.3{ }^{\circ} \mathrm{C}$. The initial temperature of the water differed from the saturation temperature in the series of experiments by 12,42 and $72 \mathrm{~K}$. The heating rate of the heater's metal varied in the range from $2400 \mathrm{~K} / \mathrm{s}$ to 6000 $\mathrm{K} / \mathrm{s}$. The average velocity of the water was $0.2 \mathrm{~m} / \mathrm{s}$. The values of $\tau_{\text {ind }}$ were determined based on the data delivered by the pressure sensor.

\section{Computational experiment}

The unsteady heating of the heater's wall and near-wall liquid layers was modeled in Comsol software. The mathematical model accounted for geometry and characteristics of materials used to manufacture the unit operational area. We modeled liquid movement, heat exchange in liquid, heat exchange in the heater, and heating by Joule heat of the heater [3]. This model allows us to calculate the superheated water layer thickness at high accuracy, as well as the heater temperature during impulse release of electric power. This model also takes into consideration the influence of nucleate boiling on the boundary layer. The assumptions adopted in the model hold true until the stage of bubble agglomeration, which means that the model is not suitable to simulate the interaction between the bubbles with high density of vaporization centers. Therefore, until the start of active vaporization, the model adequately describes the thermal state. Figure 3 gives a visual representation of the computational mesh, where we can notice a considerable decrease of the size of the computational element in the boundary layer area.

The laminar fluid flow problem

$$
\rho \frac{\partial \mathbf{u}}{\partial t}-\rho(\mathbf{u} \cdot \nabla) \mathbf{u}=\nabla \cdot\left[-p \mathbf{I}+\mu\left(\nabla \mathbf{u}+(\nabla \mathbf{u})^{\top}\right)\right]
$$

$$
\rho \nabla \cdot \mathbf{u}=0
$$

coupled with the heat transfer in fluid problem

$$
\rho C_{p} \frac{\partial T}{\partial t}+\rho C_{p} \mathbf{u} \cdot \nabla T=\mathrm{k} \nabla \cdot \nabla T
$$

was solved for the subcooled water flow domains. Here $T$ denotes transposition. The heat transfer in solid in presence of resistive heat

$$
\rho C_{p} \frac{\partial T}{\partial t}-\mathrm{k} \nabla \cdot \nabla T=\frac{1}{2} \mathbf{J} \cdot \mathbf{E}
$$

was coupled with the direct electric current problem

$$
\begin{gathered}
\nabla \cdot \mathbf{J}=0 \\
\mathbf{J}=\left(\sigma+\epsilon_{0} \epsilon_{r} \frac{\partial}{\partial t}\right) \mathbf{E} \\
\mathbf{E}=-\nabla V
\end{gathered}
$$

The changes in the temperature profile that took place beyond the first $50 \mathrm{~mm}$ of the heater length and the first 3 $\mathrm{mm}$ of the heater thickness, as well as the gravitation effect, were checked numerically and found negligible. The heater surface temperature measured by thermocouples showed good agreement $(<5 \%)$ with numerical modeling until the moment of the fully developed nucleate boiling.

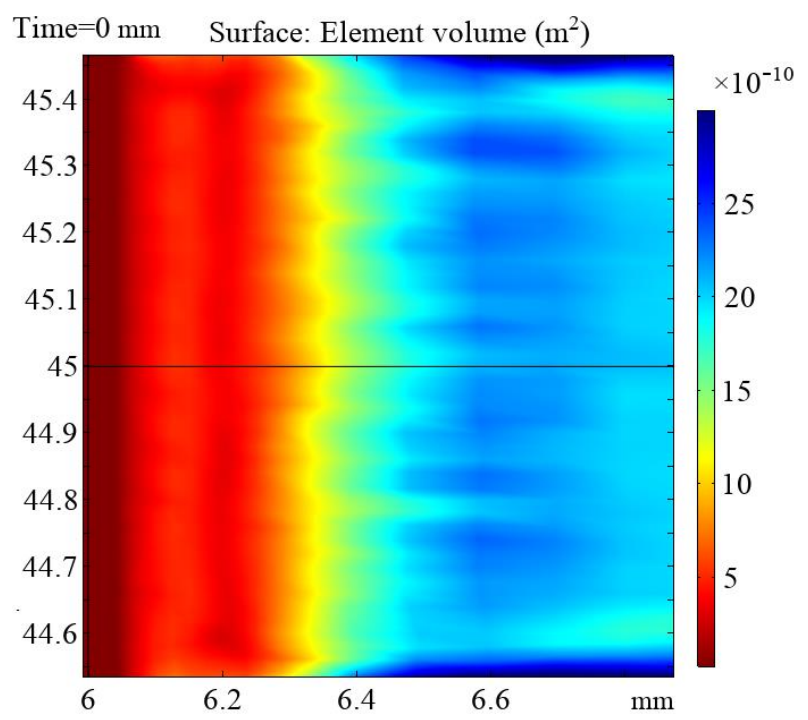

Fig. 3. Comsol computational mesh.

\section{Results and discussion}

In the stationary case, we apply the approach developed in [1] by V.I. Tolubinsky to calculate the temperature required for the start of boiling.:

$$
T_{W}=T_{S}+\left(\Delta T_{s u b} \frac{r_{C} C_{4}}{\delta}+\frac{2 \sigma T_{S}}{r \rho_{v} C_{2} r_{C}}\right) \frac{\delta}{\delta-r_{C} C_{4}}
$$

where $T_{s}$ is the saturation temperature, $\Delta T_{\text {sub }}$ is the temperature of subcooling, $\delta$ is the boundary thermal layer thickness, $r_{\mathrm{c}}$ is the active cavity radius, $r$ is a latent vaporization heat, $\rho_{\mathrm{v}}$ is the vapor density, $\sigma$ is the water surface tension coefficient.

Based on formula (1), a method for estimating the time for the start of active boiling during unsteady heating was proposed in [4]. This approach implies replacement of the the thermal layer thickness by the extrapolated layer 
thickness (Fig. 4), which increases as the initial temperature of liquid decreases. It should be noted that formula (1) and the development of this approach in [4] are based on the assumption that the temperature profile is insignificantly non-linear in the neighborhood of the heatreleasing surface.

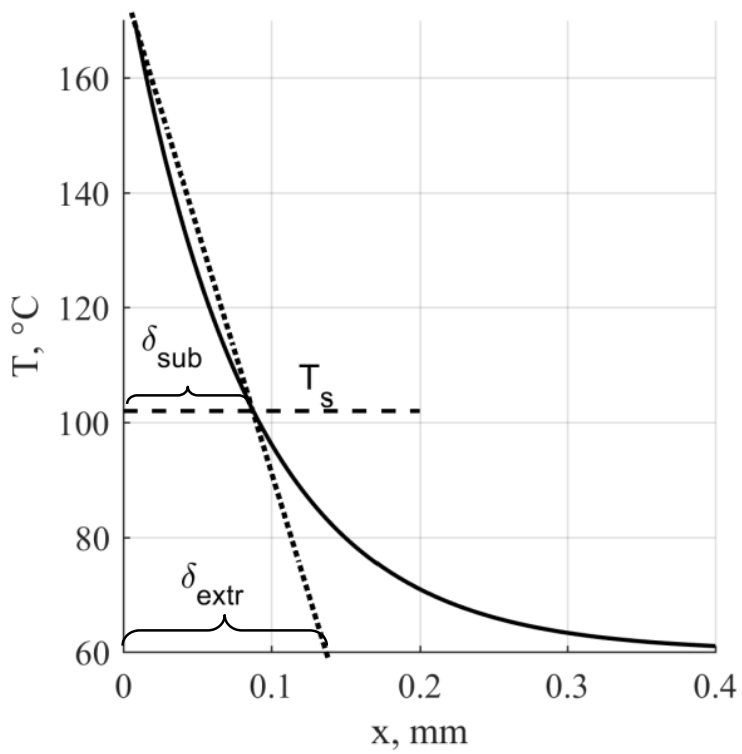

Fig. 4. Approximation scheme for the superheated liquid layer.

In [4], the thermal layer thickness in equation (8) is determined on the basis of empirical information on unsteady boiling:

$$
\delta=\beta_{1}\left(P_{0}, \Delta T_{\text {sub }}\right) \beta_{2}\left(\frac{d T_{W}}{d \tau}\right) r_{C}
$$

where $\beta_{1}$ takes into account the influence of the thermophysical properties of liquid and has the form:

$$
\beta_{1}\left(P, \Delta T_{\text {sub }}\right)=\frac{\delta_{\text {extr }}}{\delta_{\mathrm{s}}}=1.51 e^{0.008 P_{0} \Delta T_{\text {sub }}}
$$

$\beta_{2}$ is the influence of the heating rate and is set as $\beta_{2}=1$. Values of the $\beta_{1}$ and $\beta_{2}$ were determined empirically in [4]. Using $T_{w}$, the induction time until the intensive vaporization $\tau_{\text {ind }}$ can be determined by the formula

$$
\tau_{\text {ind }}=\frac{T_{w}-T_{s}+\Delta T_{s u b}}{\frac{d T_{w}}{d \tau}}
$$

Extrapolation coefficients are calculated on the basis of experimental data in the range of subcooling from 10 to 40 $\mathrm{K}$, with no forced water movement [4].

Figure 5 shows the comparison between the calculation of the induction period carried out using this technique and the experimental data obtained under forced boiling conditions. It can be seen that the calculated values significantly exceed the experimental ones. This can be explained by the presence of forced flow in experiments, which causes a significant decrease in the thermal boundary layer. This assumption is indirectly confirmed by an increase in the prediction error of the method [1], [4] for higher levels of water subcooling.

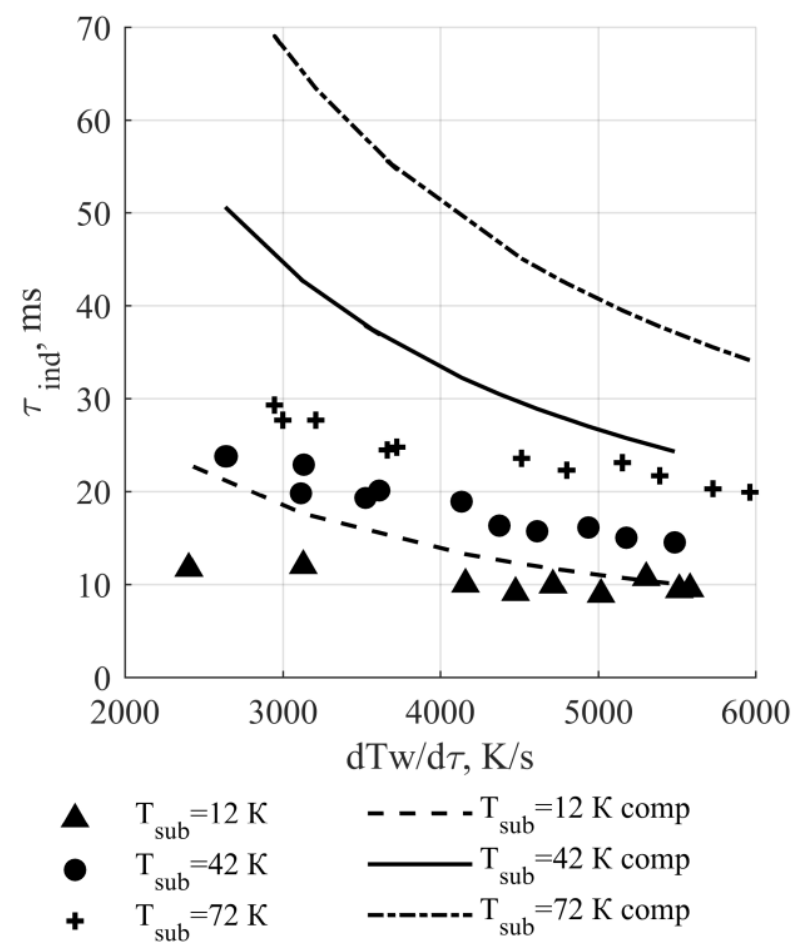

Fig. 5. Comparison of experimental results with calculations performed by the Tolubinsky-Tairov method.

By calculating in the Comsol model the heater wall temperature at the time $\tau_{\text {ind }}$, measured during the experiments, we can determine the extrapolated superheated layer thickness using formula (1). The values of the geometrical factor obtained in this way can be compared both with the induction time for intensive evaporation $\tau_{\text {ind }}$ (Fig.6) and with the superheated layer thickness $\delta_{\mathrm{s}}$, determined on the basis of the solution to the numerical model (Fig.7). It can be seen from Fig. 7 that the values of the geometric factor used to determine the induction time are inversely related to the superheated layer thickness. Note that for water subcooling of $12 \mathrm{~K}$, formula (8) allowed us to predict the start time of intensive vaporization with a satisfactory error. Therefore, taking into account the significant (5-fold) difference between the values of $\delta_{\mathrm{s}}$ and $\delta_{\text {extr }}$, we can conclude that the extrapolated superheated layer thickness in (8) has some certain specific physical meaning. 


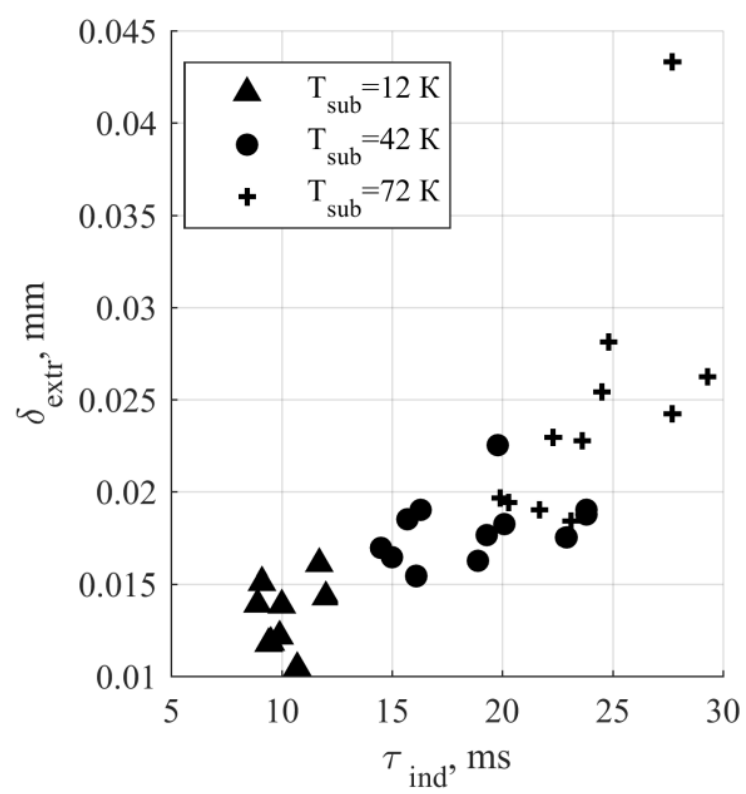

Fig. 6. Analysis of the approach from $[1,4]$ when applied to calculating $\tau_{\text {ind }}$ under conditions of forced movement

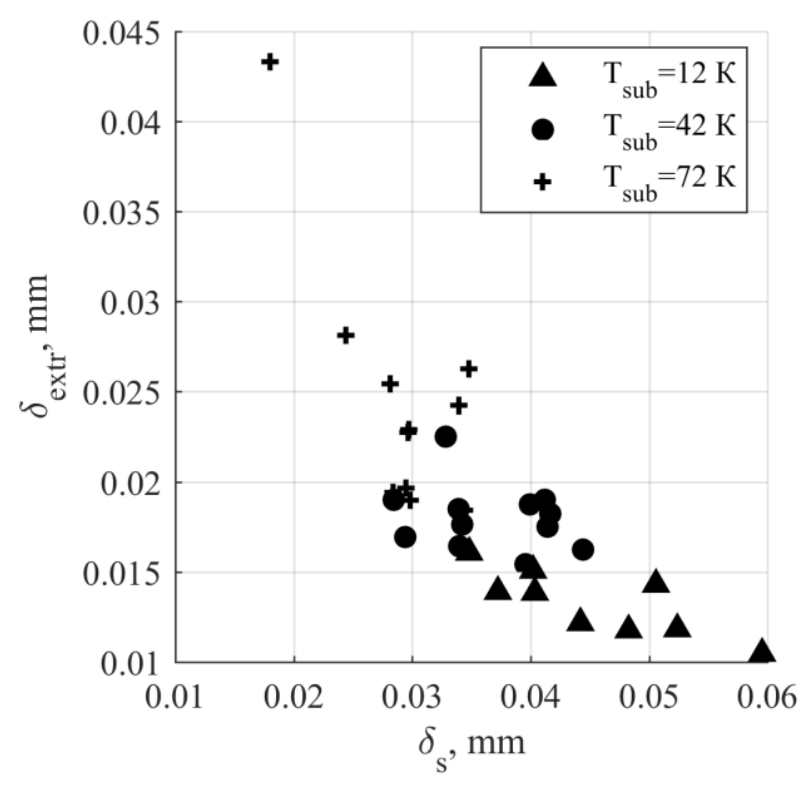

Fig. 7. Comparison between the extrapolated thickness and the superheated liquid layer thickness

\section{Conclusions}

We obtained new experimental data for the explosive boiling of a moving subcooled water flow up to the saturation temperature. We showed that the technique from [4] can be extended to unsteady heating during forced liquid flow. The characteristic warm-up thickness for explosive boiling is smaller than the superheated liquid layer thickness, which highlights the necessity to have a more detailed description of the unsteady explosive boiling of subcooled liquid.

\section{References}

1. V.I. Tolybinsky, Teploobmen pri kipenii Теплообмен при кипении, Наукова думка, 1980.

2. A. A., Levin, P. V. Khan, "Experimental observation of the maximum bubble diameter in non-stationary temperature field of subcooled boiling water flow", International Journal of Heat and Mass Transfer, 2018 vol. 124, pp.876-883. DOI:

10.1016/j.ijheatmasstransfer.2018.03.078

3. A.A. Levin., P.V. Khan "Characteristics of nucleate boiling under conditions of pulsed heat release at the heater surface", Applied Thermal Engineering. 2019. Vol.149, pp.1215-1222.

DOI: $10.1016 /$ j.applthermaleng.2018.12.126

3. E.A. Tairov, B.G. Pokusaev, M.Yu. Gritsenko. An experimental study of the onset of explosive boiling water with an increase in wall temperature // Russian National Heat Transfer Conference 3, Moscow: 2003.

\section{Acknowledgement}

This research was performed at the Melentiev Energy Systems Institute SB RAS using the equipment of the High-Temperature Circuit Multi-Access Research Center and was funded by the Russian Foundation for Basic Research, Grant No. 18-29-10019 and the Basic Research Program of the Siberian Branch of the Russian Academy of Sciences No. AAAA-A17-117030310443-5. 